Article

\title{
Comparative Analysis of Two Methods for the Detection of EGFR Mutations in Plasma Circulating Tumor DNA from Lung Adenocarcinoma Patients
}

\author{
Ming-Szu Hung ${ }^{1,2,3}$, Jr-Hau Lung ${ }^{4}$, Yu-Ching Lin 1,2,3®, Yu-Hung Fang ${ }^{1}$, Shu-Yi Huang ${ }^{1}$, \\ Yuan-Yuan Jiang ${ }^{1}$, Meng-Jer Hsieh ${ }^{1,5}$ and Ying-Huang Tsai ${ }^{5,6}$,* \\ 1 Department of Pulmonary and Critical Care Medicine, Chang Gung Memorial Hospital, Chiayi branch 61363, \\ Taiwan; m13@seed.net.tw (M.-S.H.); lin0927@cgmh.org.tw (Y.-C.L.); 8902062@cgmh.org.tw (Y.-H.F.); \\ 8802022@cgmh.org.tw (S.-Y.H.); st40339@cgmh.org.tw (Y.-Y.J.); mengjer@cgmh.org.tw (M.-J.H.) \\ 2 Department of Medicine, College of Medicine, Chang Gung University, Taoyuan 33302, Taiwan \\ 3 Department of Respiratory Care, Chang Gung University of Science and Technology, Chiayi Campus, \\ Chiayi 61363, Taiwan \\ 4 Department of Medical Research, Chang Gung Memorial Hospital, Chiayi branch 61363, Taiwan; \\ jrhaulung@gmail.com \\ 5 Department of Respiratory Care, College of Medicine, Chang Gung University, Taoyuan 33302, Taiwan \\ 6 Department of Pulmonary and Critical Care Medicine, Chang Gung Memorial Hospital, \\ Linkou branch 33305, Taiwan \\ * Correspondence: chestmed@cgmh.org.tw
}

Received: 3 May 2019; Accepted: 6 June 2019; Published: 10 June 2019

\begin{abstract}
Mutations in the epidermal growth factor receptor (EGFR) are associated with various solid tumors. This study aimed to compare two methods for the detection of EGFR mutations in circulating tumor DNA (ctDNA) from lung adenocarcinoma (LUAD) patients and to evaluate the clinical significance of EGFR mutations in ctDNA. In this prospective cohort study, the EGFR mutation status of 77 patients with stage IIIB or IV LUAD was first determined using lung cancer tissue. The amplification refractory mutation system (ARMS) and single allele base extension reaction combined with mass spectroscopy (SABER/MassARRAY) methods were also used to detect EGFR mutations in plasma ctDNA from these patients and then compared using the EGFR mutation status in lung cancer tissue as a standard. Furthermore, the relationship between the presence of EGFR mutations in ctDNA after receiving first-line EGFR-tyrosine kinase inhibitor (EGFR-TKI) therapy and survival was evaluated. The overall sensitivity and specificity for the detection of EGFR mutations in plasma ctDNA by ARMS and SABER/MassARRAY were $49.1 \%$ vs. $56 \%$ and $90 \%$ vs. $95 \%$, respectively. The agreement level between these methods was very high, with a kappa-value of 0.88 (95\% CI 0.77-0.99). Moreover, 43 of the patients who carried EGFR mutations also received first-line EGFR-TKI therapy. Notably, patients with EGFR mutations in plasma ctDNA had significantly shorter progression-free survival (9.0 months, 95\% CI 7.0-11.8, vs. 15.0 months, 95\% CI 11.7-28.2; $p=0.02$ ) and overall survival (30.6 months, 95\% CI 12.4-37.2, vs. 55.6 months, 95\% CI 25.8-61.8; $p=0.03$ ) compared to those without detectable EGFR mutations. The detection of EGFR mutations in plasma ctDNA is a promising, minimally invasive, and reliable alternative to tumor biopsy, and the presence of EGFR mutations in plasma ctDNA after first-line EGFR-TKI therapy is associated with poor prognosis.
\end{abstract}

Keywords: lung cancer; EGFR; TKI; ctDNA; ARMS; MassARRAY

\section{Introduction}

The epidermal growth factor receptor (EGFR) pathway plays an important role in the growth, proliferation, and survival of various solid tumors, including non-small cell lung cancer (NSCLC) [1]. 
EGFR is, therefore, an important potential target for lung cancer therapy. Notably, some NSCLC patients carry activating mutations in the tyrosine kinase domain of EGFR and, unlike the majority of NSCLC patients, exhibit a favorable clinical response to EGFR-tyrosine kinase inhibitor (EGFR-TKI) therapy [2].

While mutations in EGFR have been found in less than 10\% of non-Asian NSCLC patients, up to $30 \%$ of East Asian NSCLC patients carry such mutations [3]. Interestingly, most of these mutations were limited to exons 18-21 [4], and were most frequently detected in patients with lung adenocarcinoma (LUAD) [5]. Exon 19 deletions and exon 21 missense mutations are common EGFR activating mutations, and among these, exon 19 in-frame deletions and the L858R exon 21 missense mutation have been shown to represent approximately $80 \%$ of the EGFR-TKI-sensitive mutations in NSCLC [6]. Furthermore, several clinical trials have demonstrated that, in NSCLC patients, exon 19 deletions and exon 21 missense mutations were associated with a favorable response to first-line treatment with EGFR-TKIs, including gefitinib [7], erlotinib [8], and afatinib [9], compared to conventional chemotherapy. Importantly, another missense mutation, T790M in exon 20, is associated with EGFR-TKI resistance and has been detected in $30 \%-50 \%$ of the patients that initially responded to EGFR-TKI therapy but eventually acquired EGFR-TKI resistance. However, recent evidence indicates that osimertinib, a third-generation EGFR-TKI, can overcome T790M-mediated resistance to first- and second-generation EGFR-TKIs.

Liquid biopsy is a promising technique for cancer diagnosis and treatment and consists of the detection and isolation of circulating tumor cells, circulating tumor DNA (ctDNA), and exosomes as a source of genomic and proteomic information in patients with cancer [10]. In patients with lung cancer, different methods have been used successfully to detect EGFR mutations from ctDNA, and studies have demonstrated that this approach was valuable for diagnosis, predicting treatment response, and monitoring acquired therapy resistance [11,12]. While the amplification refractory mutation system (ARMS) method has been used to detect EGFR mutations in both lung cancer tissues [13] and plasma ctDNA [14], a single allele base extension reaction combined with mass spectrometry (SABER/MassARRAY) has also been used to detect the T790M EGFR mutation in plasma ctDNA [15]. However, the respective performances of the ARMS and SABER/MassARRAY methods for the clinical detection of EGFR mutations from plasma ctDNA have rarely been compared.

In this study, we determined the EGFR mutation status of LUAD patients using lung cancer tissues and compared the efficiency of the ARMS and SABER/MassARRAY methods in detecting EGFR mutations in ctDNA isolated from the plasma of these patients. The relationship between the EGFR status and clinical outcomes of LUAD patients who received first-line EGFR-TKI therapy was also evaluated.

\section{Materials and Methods}

\subsection{Patients and Study Design}

Between February 2013 and March 2017, 77 LUAD patients (57 with and 20 without EGFR mutations) were enrolled in this prospective cohort study of EGFR mutation detection in plasma ctDNA. At the start of the study, all patients were treatment-naive with stage IIIB or IV advanced LUAD, according to the 7th Edition of the American Joint Committee on Cancer (AJCC) staging system. Mutations in the EGFR gene were detected by ARMS, using the therascreen EGFR RGQ PCR kit (Qiagen, Hilden, Germany) according to the manufacturer's recommendations, or SABER/MassARRAY, using the OncoFOCUS ${ }^{\mathrm{TM}}$ Panel v1.0 (Agena Bioscience, San Diego, CA, USA) with the MassARRAY system (Agena Bioscience), as previously described [15]. The EGFR mutations examined in this study included exon 19 deletions and the T790M and L858R missense mutations.

The clinical variables including sex, age, smoking status, pathology, EGFR-TKIs, mutations, and stages of these patients were analyzed. Patients were treated with either gefitinib ( $250 \mathrm{mg} /$ day), erlotinib (150 mg/day), or afatinib (40 mg/day) until progressive disease (PD) occurred. Three months 
after treatment initiation, the response to therapy was evaluated according to the Response Evaluation Criteria in Solid Tumors (RECIST) version 1.1 [16], following examination of target lesions by a chest computed tomography (CT) scan, brain magnetic resonance imaging (MRI), or bone scan. Progression-free survival (PFS) was defined as the time from the first treatment to PD or death from any cause, and overall survival (OS) was defined as the time from the diagnosis to death from any cause or surviving patients being censored at their last follow-up.

This study was approved by the Ethics Review Board of Chang Gung Memorial Hospital (IRB No. 201600915B0 and 201101405B0).

\subsection{DNA Extraction}

For EGFR mutation status analysis in lung cancer tissues, DNA was extracted from $5 \mu \mathrm{m}$-thick sections of formalin-fixed paraffin-embedded (FFPE) tumors using the QIAamp DNA FFPE Tissue Kit (Qiagen), according to the manufacturer's recommendations.

For EGFR mutation status analysis in plasma ctDNA, blood samples were collected in ethylenediaminetetraacetic acid (EDTA) tubes after a lung cancer diagnosis or EGFR-TKI treatment failure. Then, ctDNA was purified from $1 \mathrm{~mL}$ of plasma using the QIAamp Circulating Nucleic Acid Kit (Qiagen) according to the manufacturer's recommendations.

\subsection{Statistical Analysis}

All statistical analyses were performed using the MedCalc version 15 software (MedCalc Software, Ostend, Belgium). Survival was analyzed using the Kaplan-Meier estimator and log-rank test. The agreement level between the ARMS and SABER/MassARRAY methods was evaluated using the inter-rater agreement (kappa-value) test. The difference between ARMS and SABER/MassArray was compared by pairwise comparison of receiver operating characteristic curve (ROC) curves. The difference of tumor size was compared by $t$-test. A $p$-value $<0.05$ was considered to be statistically significant.

\section{Results}

\subsection{Comparison of ARMS and SABER/MassARRAY for the Detection of EGFR Mutations in Plasma ctDNA}

In order to compare the efficiency of ARMS and SABER/MassARRAY in detecting EGFR mutations in plasma ctDNA, we first determined the EGFR mutation status of the 77 LUAD patients enrolled in our study using the ARMS method. Using DNA isolated from lung cancer tissue, EGFR mutations were detected in 57 patients, whereas the other 20 patients carried only wild-type (WT) EGFR alleles (Figure 1). Three patients in our study demonstrated uncommon EGFR mutations (2 with G719X+S768I, and 1 with S768I) and without T790M, exon 19 deletions or L858R mutations in the lung cancer tissues. Next, we used ARMS and SABER/MassARRAY to detect the presence of EGFR mutations in the ctDNA isolated from the plasma of 77 and 70 patients, respectively.

Using the EGFR mutation status established from the lung cancer tissue as the standard, the overall sensitivity, specificity, and positive predictive value (PPV) of the ARMS method were 49.1\%, $90 \%$, and $93.3 \%$, respectively (Table 1). In comparison, the overall sensitivity, specificity, and PPV of the SABER/MassARRAY method were 56\%, 95\%, and 96.6\%, respectively (Table 1). Importantly, the level of agreement between the two methods was very high, with a kappa-value of 0.88 (95\% confidence interval (CI) 0.77-0.99).

Focusing on EGFR activating mutations, the sensitivity, specificity, and PPV for the detection of exon 19 deletions by ARMS and SABER/MassARRAY were 50\% vs. 53.8\%, 95.6\% vs. 97.7\%, and $88.9 \%$ vs. 93.3\%, respectively (Table 2). For the detection of the L858R missense mutation by ARMS and SABER/MassARRAY, the sensitivity, specificity, and PPV were $45 \%$ vs. $47.4 \%, 100 \%$ vs. $100 \%$, and $100 \%$ vs. $100 \%$, respectively (Table 3 ). 


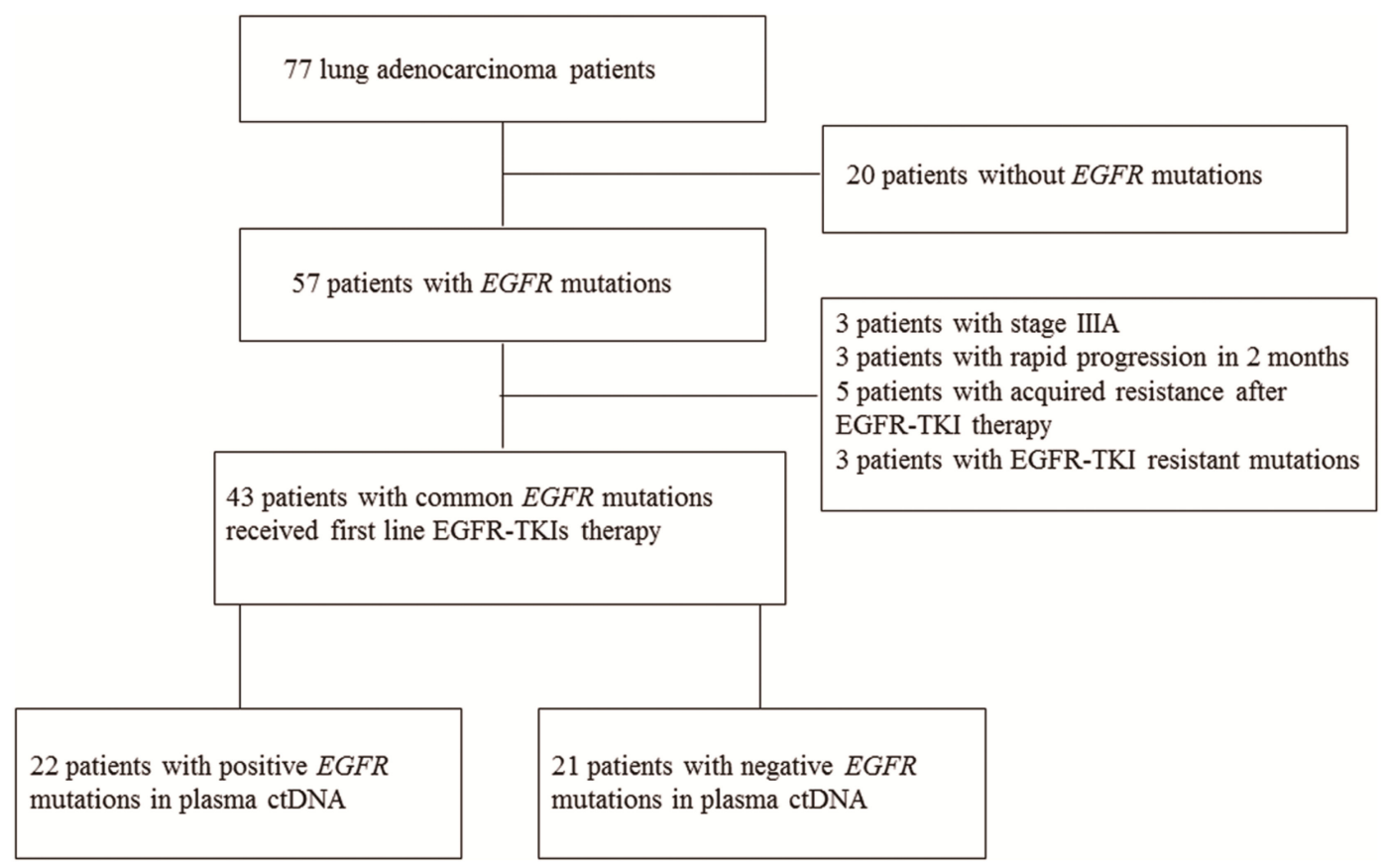

Figure 1. Flow chart of the study.

Table 1. Comparison of amplification refractory mutation system (ARMS) and single allele base extension reaction combined with mass spectroscopy (SABER/MassARRAY) methods in detecting epidermal growth factor receptor (EGFR) mutations from circulating tumor DNA (ctDNA).

\begin{tabular}{cccc}
\hline \multirow{4}{c}{ ARMS vs. Tissue } \\
\hline \multirow{2}{*}{ ARMS } & \multicolumn{3}{c}{ Tissue } \\
\cline { 2 - 4 } & Negative & Positive & \\
\cline { 2 - 4 } Negative & 2 & 29 & NPV: $48.7 \%$ \\
Positive & Specificity: $90 \%$ & PPV: $93.3 \%$ & \\
Sensitivity: $49.1 \%$ & SABER/MassARRAY vs. Tissue \\
\hline \multicolumn{5}{c}{ Tissue } \\
\hline \multirow{5}{*}{ SABER/MassARRAY } & Negative & Positive \\
\cline { 2 - 4 } & 19 & 22 & \\
\hline Negative & 1 & 28 & NPV: $46.3 \%$ \\
Positive & Specificity: $95 \%$ & PPV: $96.6 \%$ & \\
Sensitivity: $56 \%$ &
\end{tabular}

Tissue EGFR was used as the standard reference. PPV: positive predictive value; NPV: negative predictive value.

Table 2. Comparison of ARMS and SABER/MassARRAY methods in detecting exon 19 deletions EGFR mutations from ctDNA.

\begin{tabular}{|c|c|c|c|}
\hline \multicolumn{4}{|c|}{ Exon 19 Deletions ARMS vs. Tissue } \\
\hline \multirow{2}{*}{ ARMS } & \multicolumn{2}{|c|}{ Tissue } & \\
\hline & Negative & Positive & \\
\hline Negative & 43 & 16 & \\
\hline Positive & 2 & 16 & \\
\hline Sensitivity: $50 \%$ & Specificity: $95.6 \%$ & PPV: $88.9 \%$ & NPV: $72.9 \%$ \\
\hline \multicolumn{4}{|c|}{ SABER/MassARRAY vs. Tissue } \\
\hline \multirow{2}{*}{ SABER/MassARRAY } & \multicolumn{2}{|c|}{ Tissue } & \\
\hline & Negative & Positive & \\
\hline Negative & 43 & 12 & \\
\hline Positive & 1 & 14 & \\
\hline Sensitivity: $53.8 \%$ & Specificity: $97.7 \%$ & PPV: 93.3\% & NPV: $78.2 \%$ \\
\hline
\end{tabular}


Table 3. Comparison of ARMS and SABER/MassARRAY methods in detecting L858R EGFR mutations from ctDNA.

\begin{tabular}{cccc}
\hline \multirow{4}{c}{ L858R ARMS vs. Tissue } \\
\hline \multirow{2}{*}{ ARMS } & \multicolumn{3}{c}{ Tissue } \\
\cline { 2 - 4 } & Negative & Positive & \\
Negative & 0 & 11 & NPV: $83.8 \%$ \\
Positive & Specificity: $100 \%$ & PPV: $100 \%$ & \\
Sensitivity: $45 \%$ & \multicolumn{5}{c}{ SABER/MassARRAY vs. Tissue } \\
\hline \multicolumn{5}{c}{ Tissue } \\
\hline SABER/MassARRAY & \multicolumn{4}{c}{ Positive } \\
\cline { 2 - 4 } & Negative & 10 & \\
Negative & 51 & 9 & NPV: $83.6 \%$ \\
Positive & 0 & PPV: $100 \%$ & \\
Sensitivity: $47.4 \%$ & Specificity: $100 \%$ &
\end{tabular}

Tissue $E \overline{G F R}$ was used as the standard reference. PPV: positive predictive value; NPV: negative predictive value. Tissue $E G F R$ was used as the standard reference. PPV: positive predictive value; NPV: negative predictive value.

Moreover, a de novo T790M mutation, which is associated with EGFR-TKI resistance, was detected in the plasma ctDNA of one patient, while the T790M mutation was also detected in the re-biopsy samples of five patients following EGFR-TKI therapy failure. The sensitivity, specificity, and PPV for the detection of the T790M mutation by ARMS and SABER/MassARRAY were 33.3\% vs. $50 \%, 100 \%$ vs. $100 \%$, and $100 \%$ vs. $100 \%$, respectively (Table 4 ). The difference between ARMS vs. SABER/MassARRAY were compared by a pairwise comparison of ROC curves and were not statistically significant for EGFR $(p=0.0687)$, L858R $(p=1.0000)$, Exon 19 deletions $(p=0.1708)$, and $\operatorname{T790M~}(p=0.3173)$ mutations.

Table 4. Comparison of ARMS and SABER/MassARRAY methods in detecting T790M EGFR mutations from ctDNA.

\begin{tabular}{|c|c|c|c|}
\hline \multicolumn{4}{|c|}{ T790M ARMS vs. Tissue } \\
\hline \multirow{2}{*}{ ARMS } & \multicolumn{2}{|c|}{ Tissue } & \\
\hline & Negative & Positive & \\
\hline Negative & 70 & 4 & \\
\hline Positive & 0 & 2 & \\
\hline Sensitivity: $33.3 \%$ & Specificity: $100 \%$ & PPV: $100 \%$ & NPV: $94.6 \%$ \\
\hline \multicolumn{4}{|c|}{ SABER/MassARRAY vs. Tissue } \\
\hline \multirow{2}{*}{ SABER/MassARRAY } & \multicolumn{2}{|c|}{ Tissue } & \\
\hline & Negative & Positive & \\
\hline Negative & 64 & 3 & \\
\hline Positive & 0 & 3 & \\
\hline Sensitivity: $50 \%$ & Specificity: $100 \%$ & PPV: $100 \%$ & NPV: $95.5 \%$ \\
\hline
\end{tabular}

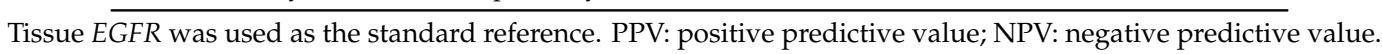

\subsection{Relationship between the EGFR Mutation Status after EGFR-TKI Therapy and Survival}

In this study, we also evaluated the correlation between the presence of common EGFR mutations and the outcomes for patients who received EGFR-TKI therapy. Out of the 77 patients enrolled in the study, 43 carried common EGFR-activating mutations (L858R missense mutation and exon 19 deletions) detected by ARMS and received first-line EGFR-TKI therapy (Table 5).

Among these 43 patients, the presence of EGFR mutations in plasma ctDNA was significantly associated with shorter progression-free survival (PFS) (9.0 months for patients with detectable EGFR mutations, $95 \%$ CI $7.0-11.8$, vs. 15.0 months for patients without detectable EGFR mutations, $95 \%$ CI 11.7-28.2; $p=0.02$ ) (Figure 2) and OS (30.6 months for patients with detectable EGFR mutations, 
95\% CI 12.4-37.2, vs. 55.6 months for patients without detectable EGFR mutations, 95\% CI 25.8-61.8; $p=0.03$ ) (Figure 3).

In addition, common EGFR mutations were detected by SABER/MassArray in 37 of the 43 patients. Among these 37 patients, the presence of EGFR mutations in plasma ctDNA was associated with an insignificant trend for shorter PFS (11.2 months for patients with detectable EGFR mutations, $95 \%$ CI 7.0-12.5, vs. 14.0 months for patients without detectable EGFR mutations, $95 \%$ CI 7.6-38.5; $p=0.28$ ) (Figure S1) and OS (30.6 months for patients with detectable EGFR mutations, 95\% CI 12.4-61.8, vs. 35.7 months for patients without detectable EGFR mutations, 95\% CI 17.3-61.8; $p=0.71$ ) (Figure S2).

Table 5. Clinical characteristics of first-line EGFR-tyrosine kinase inhibitor (EGFR-TKI) treatment patients.

\begin{tabular}{cc}
\hline Characteristics & No (\%) \\
\hline Patient & $43(100)$ \\
Sex & $15(34.9)$ \\
Male & $28(65.1)$ \\
Female & \\
Smoking & $4(9.3)$ \\
Yes & $39(90.7)$ \\
No & 71 \\
Age (year)(median) & \\
Pathology & $43(100)$ \\
Adenocarcinoma & \\
EGFR-TKI & $20(46.5)$ \\
Gefitinib & $13(30.2)$ \\
Erlotinib & $10(23.3)$ \\
Afatinib & \\
Mutations & $27(62.8)$ \\
Exon 19 & $16(37.2)$ \\
Exon 21 & \\
Stage & $3(7)$ \\
IIIB & $40(93)$ \\
IV &
\end{tabular}

No: number.

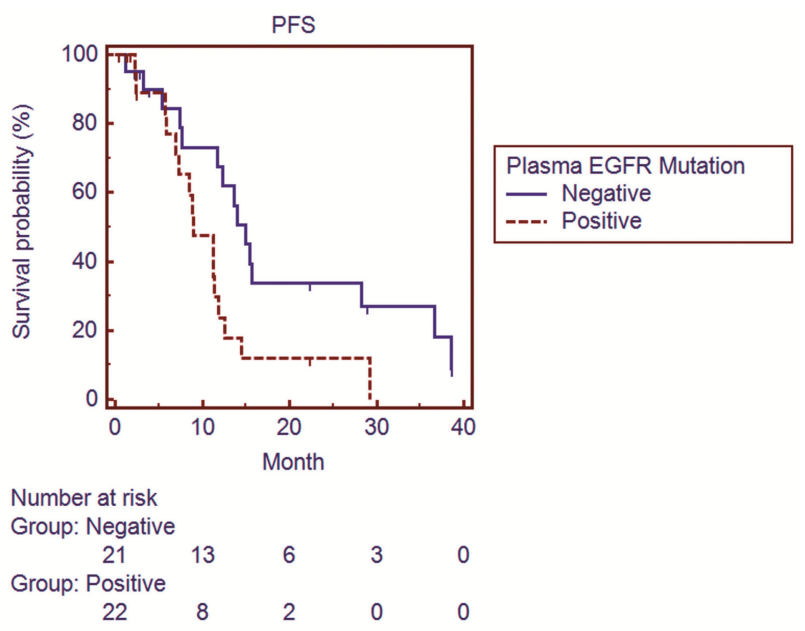

Figure 2. Survival curves showing the progression-free survival (PFS) of lung adenocarcinoma patients with and without detectable epidermal growth factor receptor (EGFR) mutations in plasma circulating tumor DNA (ctDNA) by ARMS.

Blood was collected from nine patients upon the diagnosis of lung cancer and 34 patients after EGFR-TKI therapy. The sizes of tumors at the time of the detection of plasma EGFR mutations were also 
evaluated. In 43 patients, regarding prognosis evaluation, the size of tumors at the time of the detection of EGFR mutations from plasma ctDNA were measurable using chest CT in 34 patients for ARMS and 30 patients for SABER/MassARRAY. Among these 34 patients, 12 patients showed decreased tumor size, 15 patients showed unchanged tumor size and, 7 patients showed increased tumor size at the time of blood collection. The average tumor size was significantly larger in patients with detectable plasma EGFR mutations compared to those without detectable plasma EGFR mutations (ARMS, $62.8 \mathrm{~mm}, 95 \%$ CI 43.1-82.5 mm vs. $32.8 \mathrm{~mm}, 95 \%$ CI 22.3-43.2 mm, $p<0.05$; SABER/MassARRAY, $62.1 \mathrm{~mm}, 95 \%$ CI 40.3-83.9 mm vs. $31.4 \mathrm{~mm}, 95 \%$ CI 20.2-42.6 mm, $p<0.05$ ) (Figure S3A,B).

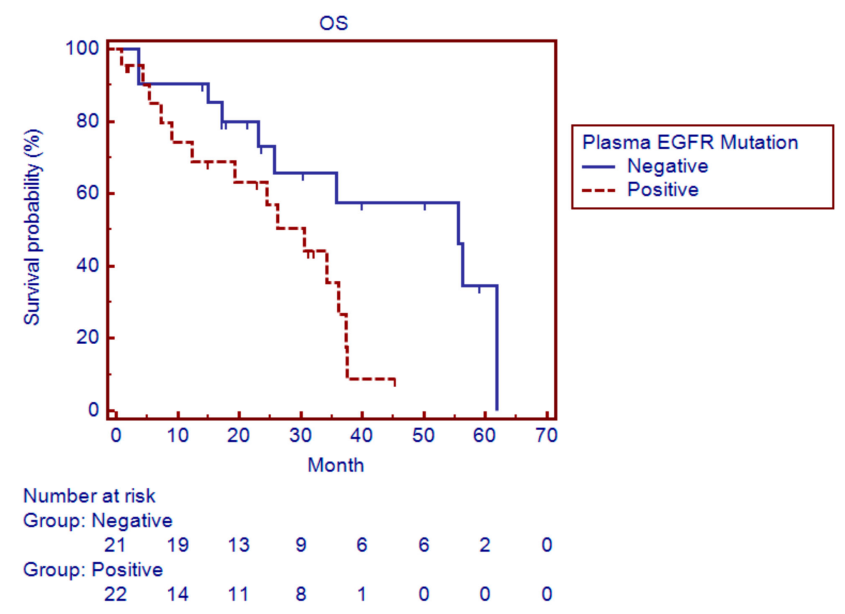

Figure 3. Survival curves showing the overall survival (OS) of lung adenocarcinoma patients with and without detectable epidermal growth factor receptor (EGFR) mutations in plasma ctDNA by ARMS.

\section{Discussion}

In this study, we demonstrated that both ARMS and SABER/MassARRAY are efficient methods for the detection of EGFR mutations in plasma ctDNA of LUAD patients. Furthermore, the two methods showed great potential for the clinical assessment of LUAD patients, exhibiting strong inter-rater agreement, high specificity, and high PPV. Our analyses also indicated that the presence of EGFR mutations in plasma ctDNA after first-line EGFR-TKI therapy was associated with poor prognosis.

A Qiagen therascreen EGFR RGQ PCR kit (ARMS method) has been approved in the United States as well as Europe and Asian countries to detect EGFR mutations with high sensitivity and specificity in lung cancer tissues [13]. Our results demonstrated that this kit could also be used reliably to detect EGFR mutations in plasma ctDNA isolated from lung cancer patients. Importantly, in our study, the observed sensitivity and specificity of ARMS were comparable to those reported in previous studies (sensitivity between $43.1 \%$ and $85.7 \%$, with high specificity) $[14,17,18]$. Although our observed sensitivity for ARMS (49.1\%) might appear somewhat lower than in these reports, this could be due to our study design, where blood samples were collected after EGFR-TKI therapy. Indeed, a previous study has reported that the detection rate of EGFR mutations in plasma ctDNA from lung cancer patients was lower after chemotherapy or EGFR-TKI therapy [19].

The MassARRAY system is a medium-throughput multiplexed ultrasensitive mutation detection system based on matrix-assisted laser desorption ionization time-of-flight (MALDI-TOF). MassARRAY has been successfully used for the detection of mutations in plasma ctDNA from patients with solid tumors, and the reported limit-of-detection was $0.1 \%$ ctDNA [20]. Furthermore, a sensitivity of $61 \%$ has been reported using MassARRAY to detect EGFR mutations in plasma ctDNA from lung cancer patients [21], while the SABER/MassARRAY method has been successfully used to detect the T790M EGFR mutation in EGFR-TKI refractory lung cancer patients [15]. In this study, we further demonstrated that SABER/MassARRAY was a reliable and sensitive method for the detection of exon 19 deletions and L858R EGFR mutations in addition to the T790M mutation in plasma ctDNA. Although 
comparable, the observed sensitivity of SABER/MassARRAY was slightly higher than that of ARMS (56\% vs. $49.1 \%$ ). In our study, SABER/MassARRAY also exhibited higher sensitivity compared to ARMS (50\% vs. 33\%) for the detection of the T790M mutation associated with acquired EGFR-TKI resistance. In both cases, the trend of higher sensitivity of SABER/MassARRAY, compared to ARMS, could be related to its lower limit-of-detection for mutations in ctDNA $(0.1 \%$ for SABER/MassARRAY vs. $1 \%$ for ARMS) [22]. In our study, false positive results were observed in exon 19 deletions (two patients in ARMS, and one patient in SABER/MassArray). The reasons for positive results may be due to heterogeneity of the tumor, the site of the biopsy, and timing of the biopsy.

In this study, we also found that the presence of EGFR mutations in plasma ctDNA detected by ARMS after EGFR-TKI therapy was associated with decreased PFS and OS. Importantly, a previous study also reported a decreased rate of EGFR mutations in ctDNA after EGFR-TKI therapy, which was associated with the disease status in lung cancer patients [23]. In our study, the presence of EGFR mutations in plasma ctDNA was also associated with a larger tumor size. Therefore, our findings further confirm that the presence of EGFR mutations after EGFR-TKI therapy is an adverse prognostic marker in LUAD patients with common EGFR activating mutations at diagnosis. In the SABER/MassARRAY group, only trends toward shorter PFS and OS were observed in patients with the presence of EGFR mutations in plasma ctDNA, which may be due to the lower patient number. In addition to ARMS and SABER/MassARRAY, another novel method for the detection of EGFR mutations in plasma ctDNA is droplet digital PCR (ddPCR) and next-generation sequencing (NGS) (Table S1). Based on the compartmentalization and amplification of single DNA molecules, ddPCR is generally accepted as the most sensitive method for the detection and quantification of EGFR mutations in plasma ctDNA (71\%-100\% for EGFR-activating mutations) [24]. However, in most medical institutions, the use of ddPCR is limited, which is mainly due to the need for specialized equipment and its higher cost in terms of reagents and labor. NGS enables the detection of multiple genetic alterations simultaneously, and has been accepted as a noninvasive tool for the identification and monitoring of cancer mutations. In advanced-stage lung cancer patients, the overall sensitivity of NGS in detecting EGFR mutations from plasma ctDNA has been reported to be between $60.9 \%$ and $82.1 \%$ [25]. Nevertheless, NGS methods are still relatively expensive and time-consuming [26]. Therefore, our results further demonstrate that, in addition to their use in detecting EGFR mutations in tissue samples, both ARMS and MassARRAY currently represent reliable alternatives to ddPCR and NGS for the detection of EGFR mutations in plasma ctDNA.

\section{Conclusions}

In conclusion, our study showed that the detection of EGFR mutations in plasma ctDNA using either ARMS or SABER/MassARRAY is a promising, minimally invasive, and reliable alternative to tumor biopsy. Moreover, our data indicated that as part of the treatment follow-up, monitoring EGFR mutation status in plasma ctDNA could be used as a prognostic marker for LUAD patients carrying common EGFR-activating mutations and receiving first-line EGFR-TKI therapy.

Supplementary Materials: The following are available online at http:/www.mdpi.com/2072-6694/11/6/803/ s1. Table S1: Comparison of platforms for detection of EGFR mutations from plasma ctDNA; Figure S1: Survival curves showing the progression-free survival (PFS) of lung adenocarcinoma patients with and without detectable epidermal growth factor receptor (EGFR) mutations in plasma circulating tumor DNA (ctDNA) by SABER/MassARRAY; Figure S2: Survival curves showing the overall survival (OS) of lung adenocarcinoma patients with and without detectable epidermal growth factor receptor (EGFR) mutations in plasma circulating tumor DNA (ctDNA) by SABER/MassARRAY; Figure S3: The average tumor size in patients with detectable plasma EGFR mutations compared to those without detectable plasma EGFR mutations. A. ARMS, B. SABER/MassARRAY.

Author Contributions: Conceptualization, M.-S.H., J.-H.L., Y.-C.L., Y.-H.F., M.-J.H. and Y.-H.T.; Data curation, J.-H.L. and Y.-C.L.; Formal analysis, Y.-H.F. and S.-Y.H.; Funding acquisition, M.-S.H., Y.-C.L. and Y.-H.T.; Investigation, M.-S.H., Y.-C.L., Y.-H.F. and M.-J.H.; Methodology, J.-H.L., Y.-C.L. and Y.-Y.J.; Project administration, M.-S.H., J.-H.L., Y.-H.F., S.-Y.H. and Y.-Y.J.; Resources, Y.-Y.J. and Y.-H. T.; Software, M.-S.H.; Supervision, Y.-H.T.; Validation, M.-S.H., J.-H.L. and M.-J.H.; Writing—original draft, M.-S.H., J.-H.L., Y.-C.L., Y.-H. F., S.-Y.H., Y.-Y. J. and M.-J.H.; Writing-review \& editing, M.-S.H., J.-H.L. and Y.-H.T. 
Funding: This study was supported by grants CORPG6B0353, CORPG6B0363, CORPG6F0042, and CORPG6F0041 from the Chang Gung Memorial Hospital, Chiayi, Taiwan (R.O.C.).

Acknowledgments: We would like to acknowledge the single allele base extension reaction combined with mass spectroscopy (SABER/MassARRAY) service provided by the Feng Chi Biothech Corp (Taipei, Taiwan).

Conflicts of Interest: The authors declare no conflict of interest.

\section{References}

1. Arteaga, C.L. Epidermal growth factor receptor dependence in human tumors: More than just expression? Oncologist 2002, 7, S31-S39. [CrossRef] [PubMed]

2. Lynch, T.J.; Bell, D.W.; Sordella, R.; Gurubhagavatula, S.; Okimoto, R.A.; Brannigan, B.W.; Harris, P.L.; Haserlat, S.M.; Supko, J.G.; Haluska, F.G.; et al. Activating mutations in the epidermal growth factor receptor underlying responsiveness of non-small-cell lung cancer to gefitinib. N. Engl. J. Med. 2004, 350, 2129-2139. [CrossRef] [PubMed]

3. Wu, Y.L.; Zhong, W.Z.; Li, L.Y.; Zhang, X.T.; Zhang, L.; Zhou, C.C.; Liu, W.; Jiang, B.; Mu, X.L.; Lin, J.Y.; et al. Epidermal growth factor receptor mutations and their correlation with gefitinib therapy in patients with non-small cell lung cancer: A meta-analysis based on updated individual patient data from six medical centers in mainland China. J. Thorac. Oncol. 2007, 2, 430-439. [CrossRef] [PubMed]

4. Paez, J.G.; Janne, P.A.; Lee, J.C.; Tracy, S.; Greulich, H.; Gabriel, S.; Herman, P.; Kaye, F.J.; Lindeman, N.; Boggon, T.J.; et al. EGFR mutations in lung cancer: Correlation with clinical response to gefitinib therapy. Science 2004, 304, 1497-1500. [CrossRef] [PubMed]

5. Huang, S.F.; Liu, H.P.; Li, L.H.; Ku, Y.C.; Fu, Y.N.; Tsai, H.Y.; Chen, Y.T.; Lin, Y.F.; Chang, W.C.; Kuo, H.P.; et al. High frequency of epidermal growth factor receptor mutations with complex patterns in non-small cell lung cancers related to gefitinib responsiveness in Taiwan. Clin. Cancer Res. 2004, 10, 8195-8203. [CrossRef] [PubMed]

6. Gazdar, A.F.; Shigematsu, H.; Herz, J.; Minna, J.D. Mutations and addiction to EGFR: The Achilles 'heal' of lung cancers? Trends Mol. Med. 2004, 10, 481-486. [CrossRef] [PubMed]

7. Mok, T.S.; Wu, Y.L.; Thongprasert, S.; Yang, C.H.; Chu, D.T.; Saijo, N.; Sunpaweravong, P.; Han, B.; Margono, B.; Ichinose, Y.; et al. Gefitinib or carboplatin-paclitaxel in pulmonary adenocarcinoma. N. Engl. J. Med. 2009, 361, 947-957. [CrossRef]

8. Zhou, C.; Wu, Y.L.; Chen, G.; Feng, J.; Liu, X.Q.; Wang, C.; Zhang, S.; Wang, J.; Zhou, S.; Ren, S.; et al. Erlotinib versus chemotherapy as first-line treatment for patients with advanced EGFR mutation-positive non-small-cell lung cancer (OPTIMAL, CTONG-0802): A multicentre, open-label, randomised, phase 3 study. Lancet Oncol. 2011, 12, 735-742. [CrossRef]

9. Wu, Y.L.; Zhou, C.; Hu, C.P.; Feng, J.; Lu, S.; Huang, Y.; Li, W.; Hou, M.; Shi, J.H.; Lee, K.Y.; et al. Afatinib versus cisplatin plus gemcitabine for first-line treatment of Asian patients with advanced non-small-cell lung cancer harbouring EGFR mutations (LUX-Lung 6): An open-label, randomised phase 3 trial. Lancet Oncol. 2014, 15, 213-222. [CrossRef]

10. Palmirotta, R.; Lovero, D.; Cafforio, P.; Felici, C.; Mannavola, F.; Pelle, E.; Quaresmini, D.; Tucci, M.; Silvestris, F. Liquid biopsy of cancer: A multimodal diagnostic tool in clinical oncology. Ther. Adv. Med. Oncol. 2018, 10. [CrossRef]

11. Douillard, J.Y.; Ostoros, G.; Cobo, M.; Ciuleanu, T.; Cole, R.; McWalter, G.; Walker, J.; Dearden, S.; Webster, A.; Milenkova, T.; et al. Gefitinib treatment in EGFR mutated caucasian NSCLC: Circulating-free tumor DNA as a surrogate for determination of EGFR status. J. Thorac. Oncol. 2014, 9, 1345-1353. [CrossRef] [PubMed]

12. Marchetti, A.; Palma, J.F.; Felicioni, L.; de Pas, T.M.; Chiari, R.; del Grammastro, M.; Filice, G.; Ludovini, V.; Brandes, A.A.; Chella, A.; et al. Early Prediction of Response to Tyrosine Kinase Inhibitors by Quantification of EGFR Mutations in Plasma of NSCLC Patients. J. Thorac. Oncol. 2015, 10, 1437-1443. [CrossRef] [PubMed]

13. Vallee, A.; le Loupp, A.G.; Denis, M.G. Efficiency of the Therascreen(R) RGQ PCR kit for the detection of EGFR mutations in non-small cell lung carcinomas. Clin. Chim. Acta 2014, 429, 8-11. [CrossRef]

14. Kimura, H.; Suminoe, M.; Kasahara, K.; Sone, T.; Araya, T.; Tamori, S.; Koizumi, F.; Nishio, K.; Miyamoto, K.; Fujimura, M.; et al. Evaluation of epidermal growth factor receptor mutation status in serum DNA as a predictor of response to gefitinib (IRESSA). Br. J. Cancer. 2007, 97, 778-784. [CrossRef] [PubMed] 
15. Sakai, K.; Horiike, A.; Irwin, D.L.; Kudo, K.; Fujita, Y.; Tanimoto, A.; Sakatani, T.; Saito, R.; Kaburaki, K.; Yanagitani, N.; et al. Detection of epidermal growth factor receptor T790M mutation in plasma DNA from patients refractory to epidermal growth factor receptor tyrosine kinase inhibitor. Cancer Sci. 2013, 104, 1198-1204. [CrossRef]

16. Eisenhauer, E.A.; Therasse, P.; Bogaerts, J.; Schwartz, L.H.; Sargent, D.; Ford, R.; Dancey, J.; Arbuck, S.; Gwyther, S.; Mooney, M.; et al. New response evaluation criteria in solid tumours: Revised RECIST guideline (version 1.1). Eur. J. Cancer 2009, 45, 228-247. [CrossRef]

17. Wang, S.; Han, X.; Hu, X.; Wang, X.; Zhao, L.; Tang, L.; Feng, Y.; Wu, D.; Sun, Y.; Shi, Y. Clinical significance of pretreatment plasma biomarkers in advanced non-small cell lung cancer patients. Clin. Chim. Acta 2014, 430, 63-70. [CrossRef]

18. Liu, X.; Lu, Y.; Zhu, G.; Lei, Y.; Zheng, L.; Qin, H.; Tang, C.; Ellison, G.; McCormack, R.; Ji, Q. The diagnostic accuracy of pleural effusion and plasma samples versus tumour tissue for detection of EGFR mutation in patients with advanced non-small cell lung cancer: Comparison of methodologies. J. Clin. Pathol. 2013, 66, 1065-1069. [CrossRef]

19. Zhang, C.; Wei, B.; Li, P.; Yang, K.; Wang, Z.; Ma, J.; Guo, Y. Prognostic value of plasma EGFR ctDNA in NSCLC patients treated with EGFR-TKIs. PLoS ONE 2017, 12, e0173524. [CrossRef]

20. Mehrotra, M.; Singh, R.R.; Loghavi, S.; Duose, D.Y.; Barkoh, B.A.; Behrens, C.; Patel, K.P.; Routbort, M.J.; Kopetz, S.; Broaddus, R.R.; et al. Detection of somatic mutations in cell-free DNA in plasma and correlation with overall survival in patients with solid tumors. Oncotarget 2018, 9, 10259-10271. [CrossRef]

21. Brevet, M.; Johnson, M.L.; Azzoli, C.G.; Ladanyi, M. Detection of EGFR mutations in plasma DNA from lung cancer patients by mass spectrometry genotyping is predictive of tumor EGFR status and response to EGFR inhibitors. Lung Cancer 2011, 73, 96-102. [CrossRef]

22. Ellison, G.; Donald, E.; McWalter, G.; Knight, L.; Fletcher, L.; Sherwood, J.; Cantarini, M.; Orr, M.; Speake, G. A comparison of ARMS and DNA sequencing for mutation analysis in clinical biopsy samples. J. Exp. Clin. Cancer Res. 2010, 29, 132. [CrossRef] [PubMed]

23. Mok, T.; Wu, Y.L.; Lee, J.S.; Yu, C.J.; Sriuranpong, V.; Sandoval-Tan, J.; Ladrera, G.; Thongprasert, S.; Srimuninnimit, V.; Liao, M.; et al. Detection and Dynamic Changes of EGFR Mutations from Circulating Tumor DNA as a Predictor of Survival Outcomes in NSCLC Patients Treated with First-line Intercalated Erlotinib and Chemotherapy. Clin. Cancer Res. 2015, 21, 3196-3203. [CrossRef] [PubMed]

24. Thress, K.S.; Brant, R.; Carr, T.H.; Dearden, S.; Jenkins, S.; Brown, H.; Hammett, T.; Cantarini, M.; Barrett, J.C. EGFR mutation detection in ctDNA from NSCLC patient plasma: A cross-platform comparison of leading technologies to support the clinical development of AZD9291. Lung Cancer 2015, 90, 509-515. [CrossRef]

25. Uchida, J.; Kato, K.; Kukita, Y.; Kumagai, T.; Nishino, K.; Daga, H.; Nagatomo, I.; Inoue, T.; Kimura, M.; Oba, S.; et al. Diagnostic Accuracy of Noninvasive Genotyping of EGFR in Lung Cancer Patients by Deep Sequencing of Plasma Cell-Free DNA. Clin. Chem. 2015, 61, 1191-1196. [CrossRef] [PubMed]

26. Elazezy, M.; Joosse, S.A. Techniques of using circulating tumor DNA as a liquid biopsy component in cancer management. Comput. Struct. Biotechnol. J. 2018, 16, 370-378. [CrossRef] [PubMed]

(C) 2019 by the authors. Licensee MDPI, Basel, Switzerland. This article is an open access article distributed under the terms and conditions of the Creative Commons Attribution (CC BY) license (http://creativecommons.org/licenses/by/4.0/). 